\title{
STRATEGIC APPROACHES TO THE SYNTHESIS, CHARACTERIZATION AND BIOLOGICAL EVALUATION OF SUBSTITUTED PIPERAZINYL-IMIDAZOLE CONJUGATES
}

\author{
Jyoti Gupta, Afreen Inam and Amir Azam ${ }^{凶}$ \\ Department of Chemistry, Jamia Millia Islamia, New Delhi-110025, India \\ ${ }^{\square}$ Corresponding Author: amir_sumbul@yahoo.co.in
}

\begin{abstract}
A series of hybrid molecules bearing 5-nitroimidazole linked with substituted piperazine molecules have been synthesized. The cytotoxicity of all the synthesized compounds has been evaluated on HEK293 cells with the help of MTT assay. It was found that the treatment of these compounds shows a variable toxicity profile for HEK293 cells. The results of cytotoxicity showed that in the tested concentration range, compound NJ3, NJ5, NJ8 and NJ10 show considerable cytotoxicity towards HEK293 cells.

Keywords: Piperazine, Nitroimidazole, Cell Viability Assay, MTT Assay, Cytotoxicity

RASĀYAN J. Chem., Vol. 14, No.1, 2021
\end{abstract}

\section{INTRODUCTION}

Nitroimidazole is a class of heterocyclic compounds where the nitro group is placed at position 5 of the imidazole ring. They are well known for antimycobacterial action through the bioreduction mechanism and have been found to show resistance against protozoal. They synthesize to metabolize which prevents intracellular bacterial synthesis ${ }^{1}$. There has been a quest among the chemical fraternity to find a new alternative of this drug that has a minimum side effect and at the same time uses a similar nitroimidazole motif ${ }^{2}$. Further, 5-nitroimidazole has proven bioactivity and toxicity concerning FABH enzyme. FABH has evolved as a major potential source for new antibacterial drug discovery. Since nitroimidazole compounds can get fully absorbed even through oral administration, it is much effective in a bacterial infection based on its action. Nitroimidazole has gained interest among researchers for its peculiar characteristic of penetrating deep with bacteria and getting completely submerged. ${ }^{3}$ Piperazine plays a dominant role in making tiny molecular therapeutic compounds. It is also one of the most common nitrogen-bearing heterocycles, which form the core of most approved pharmaceutical drug ${ }^{4}$. It has been reported that 51 out 1175 drugs duly approved by FDA contain piperazines ${ }^{5}$. Piperazine in the current scenario is the key constituent of drug discovery with the highest number of biological actions reported as a heterocyclic compound.

Moreover, piperazine is the most important content of therapeutical molecule, which has a remarkable binding property where it can form motifs to select ligands in the different biological mechanisms. Further, piperazine scaffolds and their associated derivatives have formed renowned pharmacophores, which are present in numerous biologically potential compounds and are proven antifungal, antibacterial, anti-psychotic, anti-HIV, anti-malarial and antidepressant agents. ${ }^{6} \mathrm{We}$ have designed a hybrid molecule bearing 5-nitroimidazole linked with substituted piperazine molecules. The diagrammatical model of the designed hybrid is shown in Fig.-1.

\section{Material and Methods}

\section{EXPERIMENTAL}

Prerequisite chemicals, reagents other necessary raw materials were procured from Merck and Aldrich chemical company, USA. The thin-layer chromatography (TLC) required fully coated aluminium sheets (Silica gel 60 F254, Merck Germany) viewed under UV light for specific marks. Veego instrument model no. REC-22038 A2 is specifically used to track melting points. Further, the Elementar Vario analyzer has 
been used to record elemental observation, which gave results with $\pm 0.4 \%$ variation of standard value. Bruker FT-IR spectrophotometer was deployed for the IR spectrum. Bruker Spectrospin DPX $300 \mathrm{MHz}$ gave a reading of $1 \mathrm{H}$, while Bruker Spectrospin DPX $100 \mathrm{MHz}$ showed 13C NMR reading. In this NMR, $\mathrm{CDCl}_{3}$ worked like a solvent while trimethylsilane worked as a local standard. In this method, the splitting trend has been designed as follows: $s$, singlet; $d$, doublet; $t$, triplet; $m$, multiplet. The values of the chemical shift have been recorded in ppm while mass spectrum by ESI-MS (AB-Sciex 2000, Applied Biosystems. All the values observed have been provided in the experimental segment.

Standard Procedure for the Preparation of Different Substituted Chloro acetamides (J1-J11)

These compounds have been synthesized by the reported method in our previous research. ${ }^{7}$

Standard Procedure for the Synthesis of Piperazinyl-imidazole ethenone (NJ1-NJ11)

The method of synthesized compounds has been reported in our earlier research. ${ }^{7}$

1-[4-(2,3-dichlorophenyl)piperazin-1-yl]-2-(5-methyl-2-nitro-1 H-imidazol-1-yl)ethanone(NJ-1)

Pale yellow color, solid (yield:96\%); mp:132 ${ }^{\circ}$; ${ }^{1} \mathrm{HNMR}\left(300 \mathrm{MHz}, \mathrm{CDCl}_{3}, \delta \mathrm{ppm}\right)=7.73(\mathrm{~s}, 1 \mathrm{H}), 7.27-$ $7.16(\mathrm{~m}, \mathrm{~J}=5.5 \mathrm{~Hz}, 2 \mathrm{H}), 6.96-6.94(\mathrm{~m}, \mathrm{~J}=1 \mathrm{~Hz}, 1 \mathrm{H}), 4.84(\mathrm{~s}, 2 \mathrm{H}), 3.83-3.70(\mathrm{~m}, \mathrm{~J}=6.5 \mathrm{~Hz}, 4 \mathrm{H}), 3.13-2.95(\mathrm{~m}$, $\mathrm{J}=9 \mathrm{~Hz}, 4 \mathrm{H}), 2.377(\mathrm{~s}, 3 \mathrm{H}) ;{ }^{13} \mathrm{C} \mathrm{NMR}\left(75 \mathrm{MHz}, \mathrm{CDCl}_{3}, \delta \mathrm{ppm}\right)=163.05,150.08,146.37,145.88,134.31$, 127.82, 127.71, 125.64, 121.39, 118.88, 51.39, 50.88, 47.92, 45.26, 42.78, 13.01; IR $(\mathrm{KBr}) v_{\max }=$ 1657(C=O), 1539(N=O), 3142(C=C), 2907(C-C), 782, 668(C-Cl) $\mathrm{cm}^{-1}$; ESI-MS $(\mathrm{m} / \mathrm{z}): 398.2[\mathrm{M}+1]^{+}$; Anal. Calcd for $\mathrm{C}_{16} \mathrm{H}_{17} \mathrm{~N}_{5} \mathrm{O}_{3} \mathrm{Cl}_{2}$ : C 48.25, H 4.30, N 17.59; Found: C 48.27, H 4.27, N 17.48\%.

1-[4-(3-chlorophenyl)piperazin-1-yl]-2-(5-methyl-2-nitro-1H-imidazol-1-yl)ethanone(NJ-2)

Pale yellow color, solid (yield:95\%); mp: $123{ }^{\circ} \mathrm{C}$; ${ }^{1} \mathrm{HNMR}\left(300 \mathrm{MHz}, \mathrm{CDCl}_{3}, \delta \mathrm{ppm}\right)=7.69(\mathrm{~s}, 1 \mathrm{H}), 7.26-$ $7.19(\mathrm{~m}, \mathrm{~J}=3.5 \mathrm{~Hz}, 2 \mathrm{H}), 6.90-6.79(\mathrm{~m}, \mathrm{~J}=5.5 \mathrm{~Hz}, 2 \mathrm{H}), 4.78(\mathrm{~s}, 2 \mathrm{H}), 3.81-3.66(\mathrm{~m}, \mathrm{~J}=15 \mathrm{~Hz}, 4 \mathrm{H}), 3.43-3.09$ $(\mathrm{m}, \mathrm{J}=17 \mathrm{~Hz}, 4 \mathrm{H}), 2.38(\mathrm{~s}, 3 \mathrm{H}) ;{ }^{13} \mathrm{C} \mathrm{NMR}\left(75 \mathrm{MHz}, \mathrm{CDCl}_{3}, \delta \mathrm{ppm}\right)=168.63,156.51,150.86,139.54$, $135.07,127.11,124.70,120.91,119.22,53.49,52.75,49.22,46.72,45.42,44.59,17.74$; IR (KBr) $v_{\max }=$ 1647(C=O), 1539(N=O), 3163(C=C), 2923(C-C) 676(C-Cl) $\mathrm{cm}^{-1}$; ESI-MS $(\mathrm{m} / \mathrm{z}): 364.2[\mathrm{M}+1]^{+}$; Anal. Calcd for $\mathrm{C}_{16} \mathrm{H}_{18} \mathrm{~N}_{5} \mathrm{O}_{3} \mathrm{Cl}$ : C 52.82, H 4.99, N 19.25; Found: C 52.78, H 4.96, N 19.45\%.

\section{1-[4-(2-methoxyphenyl)piperazin-1-yl]-2-(5-methyl-2-nitro-1H-imidazol-1-yl)ethanone(NJ-3)}

Pale yellowcolor,solid (yield:93\%); mp:127 ${ }^{\circ}$; ${ }^{1} \mathrm{HNMR}\left(300 \mathrm{MHz}, \mathrm{CDCl}_{3}, \delta \mathrm{ppm}\right)=7.72(\mathrm{~s}, 1 \mathrm{H}), 7.26-$ $7.04(\mathrm{~m}, \mathrm{~J}=11 \mathrm{~Hz}, 4 \mathrm{H}), 6.97-6.89(\mathrm{~m}, \mathrm{~J}=4 \mathrm{~Hz}, 3 \mathrm{H}), 4.82(\mathrm{~s}, 2 \mathrm{H}), 3.89-3.66(\mathrm{~m}, \mathrm{~J}=11.5 \mathrm{~Hz}, 3 \mathrm{H}), 3.15-2.95$ $(\mathrm{m}, \mathrm{J}=10 \mathrm{~Hz}, 4 \mathrm{H}), 2.37(\mathrm{~s}, 3 \mathrm{H}) ;{ }^{13} \mathrm{C} \mathrm{NMR}\left(75 \mathrm{MHz}, \mathrm{CDCl}_{3}, \delta \mathrm{ppm}\right)=162.85,152.28,146.36,145.86$, $140.13,123.97,121.38,121.13,118.58,111.47,55.49,50.75,50.31,47.88,45.33,42.82,13.02$; IR (KBr) $v_{\max }=1648(\mathrm{C}=\mathrm{O}), 1538(\mathrm{~N}=\mathrm{O}), 3108(\mathrm{C}=\mathrm{C}), 2908(\mathrm{C}-\mathrm{C}), 2836\left(\mathrm{O}_{-} \mathrm{CH}_{3}\right) \mathrm{cm}^{-1} ;$ ESI-MS $(\mathrm{m} / \mathrm{z}): 360.3[\mathrm{M}+1]^{+}$; Anal. Calcd for $\mathrm{C}_{17} \mathrm{H}_{21} \mathrm{~N}_{5} \mathrm{O}_{4}$ :C 56.82, H 5.89, N 19.49; Found: C 56.66, H 5.72, N 19.51\%.

\section{1-[4-(2-hydroxyphenyl)piperazin-1-yl]-2-(2-methyl-5-nitro-1 H-imidazol-1-yl)ethanone(NJ-4)}

Brown color, solid (yield:56\%); mp: $12{ }^{\circ} \mathrm{C}$; ${ }^{1} \mathrm{HNMR}\left(300 \mathrm{MHz}, \mathrm{CDCl}_{3}, \delta \mathrm{ppm}\right)=8.43(\mathrm{~s}, 1 \mathrm{H}), 7.40-7.25$ $(\mathrm{m}, \mathrm{J}=7.5 \mathrm{~Hz}, 4 \mathrm{H}), 5.28(\mathrm{~s}, 1 \mathrm{H}), 4.70(\mathrm{~s}, 2 \mathrm{H}), 3.85-3.63(\mathrm{~m}, \mathrm{~J}=11 \mathrm{~Hz}, 4 \mathrm{H}), 3.42-3.35(\mathrm{~m}, \mathrm{~J}=3.5 \mathrm{~Hz}, 4 \mathrm{H}), 2.29$ $(\mathrm{s}, 3 \mathrm{H}) ;{ }^{13} \mathrm{C} \mathrm{NMR}\left(75 \mathrm{MHz}, \mathrm{CDCl}_{3}, \delta \mathrm{ppm}\right)=162.75,151.98,146.41,145.95,140.12,124.07,121.19$, 117.92, 55.50, 50.65, 50.33, 47.78, 44.93, 13.00; IR (KBr) $v_{\max }=1649(\mathrm{C}=\mathrm{O}), 1543(\mathrm{~N}=\mathrm{O}), 3136(\mathrm{C}=\mathrm{C})$, 2919(C-C) $\mathrm{cm}^{-1}$; ESI-MS $(\mathrm{m} / \mathrm{z}): 346.3[\mathrm{M}+1]^{+}$; Anal. Calcd for $\mathrm{C}_{16} \mathrm{H}_{19} \mathrm{~N}_{5} \mathrm{O}_{4}$ : C 55.64, H 5.55, N 20.28; Found: C 55.87, H 5.57, N 20.41\%.

\section{2-(2-methyl-5-nitro-1H-imidazol-1-yl)-1-[4-(4-nitrophenyl)piperazin-1-yl]ethanone(NJ-5)}

Yellow color, solid (yield:95\%); mp: $165^{\circ} \mathrm{C}$; ${ }^{1} \mathrm{HNMR}\left(300 \mathrm{MHz}, \mathrm{CDCl}_{3}, \delta \mathrm{ppm}\right)=8.18-8.08$ (m, $\mathrm{J}=5 \mathrm{~Hz}, 3 \mathrm{H}), 7.06-7.03(\mathrm{~m}, \mathrm{~J}=1.5 \mathrm{~Hz}, 2 \mathrm{H}), 5.18(\mathrm{~s}, 2 \mathrm{H}), 3.67-3.38(\mathrm{~m}, \mathrm{~J}=14.5 \mathrm{~Hz}, 8 \mathrm{H}), 2.26(\mathrm{~s}, 3 \mathrm{H}) ;{ }^{13} \mathrm{C}$ $\operatorname{NMR}\left(75 \mathrm{MHz}, \mathrm{CDCl}_{3}, \delta \mathrm{ppm}\right)=165.03,154.75,146.68,145.53,137.55,126.21,123.81,113.23,48.30$, 46.18, 43.81, 41.48, 40.81, 39.98, 12.99; IR $(\mathrm{KBr}) v_{\max }=1660(\mathrm{C}=\mathrm{O}), 1537(\mathrm{~N}=\mathrm{O}), 3076(\mathrm{C}=\mathrm{C}), 2916(\mathrm{C}-$ C) $\mathrm{cm}^{-1}$; ESI-MS $(\mathrm{m} / \mathrm{z}): 374.1[\mathrm{M}]^{+}$; Anal. Calcd for $\mathrm{C}_{16} \mathrm{H}_{18} \mathrm{~N}_{6} \mathrm{O}_{5}$ : C 51.33, H 4.85, N 22.45; Found: C $51.43, \mathrm{H} 4.77, \mathrm{~N} 22.33$. 


\section{RASĀYAN J. Chem.}

Vol. 14 | No. 4 |2761-2766| October- December | 2021

1-(4-acetylpiperazin-1-yl)-2-(2-methyl-5-nitro-1 $H$-imidazol-1-yl)ethanone(NJ-6)

Beige color, solid (yield:76\%); mp: $143{ }^{\circ} \mathrm{C}$; ${ }^{1} \mathrm{HNMR}\left(300 \mathrm{MHz}, \mathrm{CDCl}_{3}, \delta \mathrm{ppm}\right)=8.19(\mathrm{~s}, 1 \mathrm{H}), 5.15(\mathrm{~s}$, $2 \mathrm{H}), 3.54-3.53(\mathrm{~m}, \mathrm{~J}=0.5 \mathrm{~Hz}, 5 \mathrm{H}), 3.45-3.37 \quad(\mathrm{~m}, \mathrm{~J}=4 \mathrm{~Hz}, 3 \mathrm{H}), 2.31-2.24 \quad(\mathrm{~s}, \mathrm{~J}=3.5 \mathrm{~Hz}, 3 \mathrm{H}), 2.05-2.01$ $(\mathrm{m}, \mathrm{J}=2 \mathrm{~Hz}, 3 \mathrm{H}) ;{ }^{13} \mathrm{C}$ NMR $\left(75 \mathrm{MHz}, \mathrm{CDCl}_{3}, \delta \mathrm{ppm}\right)=168.65,163.44,145.54,121.57,47.41,45.21$, 44.12, 43.86, 20.74, 12.36 ; IR (KBr) $v_{\max }=1640(\mathrm{C}=\mathrm{O}), 1538(\mathrm{~N}=\mathrm{O}), 3115(\mathrm{C}=\mathrm{C}), 2927(\mathrm{C}-\mathrm{C}) \mathrm{cm}^{-1}$; ESIMS $(\mathrm{m} / z): 296.3[\mathrm{M}+1]^{+}$; Anal. Calcd for $\mathrm{C}_{12} \mathrm{H}_{17} \mathrm{~N}_{5} \mathrm{O}_{4}$ : C 48.81, H 5.80, N 23.72; Found: C 48.61, H 5.88, N $23.62 \%$.

\section{1-[4-(1,3-benzodioxol-5-yl)piperazin-1-yl]-2-(2-methyl-5-nitro-1 $\boldsymbol{H}$-imidazol-1-yl)ethanone(NJ-7)}

Brown color, solid (yield:79\%); mp: $182{ }^{\circ} \mathrm{C}$; ${ }^{1} \mathrm{HNMR}\left(300 \mathrm{MHz}, \mathrm{CDCl}_{3}, \delta \mathrm{ppm}\right)=7.64(\mathrm{~s}, 1 \mathrm{H}), 6.83-6.80$ $(\mathrm{m}, \mathrm{J}=1.5 \mathrm{~Hz}, 1 \mathrm{H}), 6.67-6.63(\mathrm{~s}, \mathrm{~J}=2 \mathrm{~Hz}, 2 \mathrm{H}), 6.00(\mathrm{~s}, 2 \mathrm{H}), 5.00(\mathrm{~s}, 2 \mathrm{H}), 3.71-3.60(\mathrm{~m}, \mathrm{~J}=5.5 \mathrm{~Hz}, 4 \mathrm{H}), 2.74-$ $2.59(\mathrm{~m}, \mathrm{~J}=7.5 \mathrm{~Hz}, 3 \mathrm{H}), 2.42-2.39(\mathrm{~s}, \mathrm{~J}=1.5 \mathrm{~Hz}, 3 \mathrm{H}),{ }^{13} \mathrm{C} \mathrm{NMR}\left(75 \mathrm{MHz}, \mathrm{CDCl}_{3}, \delta \mathrm{ppm}\right)=164.12,148.65$, 148.22, 146.37, 144.90, 135.21, 127.96, 121.28, 119.93, 108.86, 107.74, 101.61, 50.81, 46.37, 13.32 ; IR $(\mathrm{KBr}) v_{\max }=1606(\mathrm{C}=\mathrm{O}), \quad 1540(\mathrm{~N}=\mathrm{O}), 3155(\mathrm{C}=\mathrm{C}), 2916(\mathrm{C}-\mathrm{C}) \mathrm{cm}^{-1}$; ESI-MS $(\mathrm{m} / \mathrm{z}): 373.3[\mathrm{M}]^{+}$; Anal. Calcd for $\mathrm{C}_{17} \mathrm{H}_{19} \mathrm{~N}_{5} \mathrm{O}_{5}$ : C 54.69, H 5.13, N 18.76; Found: C 54.71, H 5.43, N $18.62 \%$.

\section{2-(2-methyl-5-nitro-1H-imidazol-1-yl)-1-[4-(pyridin-3-yl)piperazin-1-yl]ethanone(NJ-8)}

Pale yellow color, solid (yield:67\%); mp: $149{ }^{\circ} \mathrm{C} ;{ }^{1} \mathrm{HNMR}\left(300 \mathrm{MHz}, \mathrm{CDCl}_{3}, \delta \mathrm{ppm}\right)=8.21-8.19(\mathrm{~m}, 1 \mathrm{H})$, $7.75(\mathrm{~s}, 1 \mathrm{H}), 7.56-7.50(\mathrm{~m}, \mathrm{~J}=3 \mathrm{~Hz}, 1 \mathrm{H}), 6.72-6.67(\mathrm{~m}, \mathrm{~J}=2.5 \mathrm{~Hz}, 2 \mathrm{H}), 4.87(\mathrm{~s}, 2 \mathrm{H}), 3.77-3.55(\mathrm{~m}, \mathrm{~J}=10 \mathrm{~Hz}$, $8 \mathrm{H}), 2.35(\mathrm{~s}, 3 \mathrm{H}) ;{ }^{13} \mathrm{C}$ NMR $\left(75 \mathrm{MHz}, \mathrm{CDCl}_{3}, \delta \mathrm{ppm}\right)=163.23,158.70,147.99,146.23,146.02,137.83$, 121.68, 114.31, 107.39, 47.99, 45.06, 44.86, 44.63, 42.03, 12.97; IR (KBr) $v_{\max }=1651(\mathrm{C}=\mathrm{O}), 1537(\mathrm{~N}=\mathrm{O})$, 3113(C=C), 2913(C-C)cm-1; ESI-MS $(m / z): 331.2[\mathrm{M}+1]^{+}$; Anal. Calcd for $\mathrm{C}_{15} \mathrm{H}_{18} \mathrm{~N}_{6} \mathrm{O}_{3}:$ C 54.54, H 5.49, N 25.44; Found: C 54.42, H 5.43, N 25.41\%.

\section{1-(4-benzhydrylpiperazin-1-yl)-2-(2-methyl-5-nitro-1H-imidazol-1-yl)ethanone(NJ-9)}

Beige color, solid (yield:88\%); mp: $204{ }^{\circ} \mathrm{C}$; ${ }^{1} \mathrm{HNMR}\left(300 \mathrm{MHz}, \mathrm{CDCl}_{3}, \delta \mathrm{ppm}\right)=7.66-7.65$ (s, $\mathrm{J}=0.5 \mathrm{~Hz}, 1 \mathrm{H}), 7.43-7.40(\mathrm{~m}, \mathrm{~J}=1.5 \mathrm{~Hz}, 4 \mathrm{H}), 7.33-7.19(\mathrm{~m}, \mathrm{~J}=7 \mathrm{~Hz}, 6 \mathrm{H}), 4.69(\mathrm{~s}, 1 \mathrm{H}), 4.30(\mathrm{~s}, 2 \mathrm{H}), 3.65-3.49$ $(\mathrm{m}, \mathrm{J}=8 \mathrm{~Hz}, 4 \mathrm{H}), 2.49-2.46(\mathrm{~m}, \mathrm{~J}=1.5 \mathrm{~Hz}, 4 \mathrm{H}), 2.35(\mathrm{~s}, 3 \mathrm{H}) ;{ }^{13} \mathrm{C} \mathrm{NMR}\left(75 \mathrm{MHz}, \mathrm{CDCl}_{3}, \delta \mathrm{ppm}\right)=170.29$, 148.46, 146.47, 144.86,127.29, 121.28, 119.93, 100.92,58.33, 50.81, 13.32; IR (KBr) $v_{\max }=1654(\mathrm{C}=\mathrm{O})$, 1532(N=O), 3105(C=C), 2922(C-C)cm ${ }^{-1}$; ESI-MS $(\mathrm{m} / \mathrm{z}): 420.3[\mathrm{M}+1]^{+}$; Anal. Calcd for $\mathrm{C}_{23} \mathrm{H}_{25} \mathrm{~N}_{5} \mathrm{O}_{3}: \mathrm{C}$ 65.85, H 6.01, N 16.70; Found: C 65.88, H 6.23, N 16.71\%.

\section{tert-butyl 4-(2-(2-methyl-5-nitro-1H-imidazol-1-yl)acetyl)piperazine-1-carboxylate(NJ-10)}

Brown color, solid (yield:60\%); mp: $192{ }^{\circ} \mathrm{C}$; ${ }^{1} \mathrm{HNMR}\left(300 \mathrm{MHz}, \mathrm{CDCl}_{3}, \delta \mathrm{ppm}\right)=8.04(\mathrm{~s}, 1 \mathrm{H}), 5.08(\mathrm{~s}$, $2 \mathrm{H}), 3.54-3.00(\mathrm{~m}, \mathrm{~J}=27 \mathrm{~Hz}, 8 \mathrm{H}), 2.15(\mathrm{~s}, 3 \mathrm{H}), 1.73(\mathrm{~s}, 9 \mathrm{H}) ;{ }^{13} \mathrm{C} \mathrm{NMR}\left(75 \mathrm{MHz}, \mathrm{CDCl}_{3}, \delta \mathrm{ppm}\right)=167.91$, $163.25,154.35,145.97,121.58,80.72,80.31,79.79,52.85,47.95,44.72,43.11,42.25,41.66,29.64$, 28.32, 12.93; IR $(\mathrm{KBr}) r_{\max }=1661(\mathrm{C}=\mathrm{O}), 1539(\mathrm{~N}=\mathrm{O}), 3128(\mathrm{C}=\mathrm{C}), 2923(\mathrm{C}-\mathrm{C}) \mathrm{cm}^{-1}$; ESI-MS $(\mathrm{m} / \mathrm{z})$ : 353.3[M] $]^{+}$; Anal. Calcd for $\mathrm{C}_{15} \mathrm{H}_{23} \mathrm{~N}_{5} \mathrm{O}_{5}$ : C 50.98, H 6.56, N 19.82; Found: C 50.67, H 6.43, N 19.82\%.

\section{1-(4-benzylpiperazin-1-yl)-2-(2-methyl-5-nitro-1 $H$-imidazol-1-yl)ethanone(NJ-11)}

Brown color, solid (yield:67\%); mp: $143{ }^{\circ} \mathrm{C}$; ${ }^{1} \mathrm{HNMR}\left(300 \mathrm{MHz}, \mathrm{CDCl}_{3}, \delta \mathrm{ppm}\right.$ ) = 7.65 (s, 1H), 7.39-7.27 $(\mathrm{m}, \mathrm{J}=6 \mathrm{~Hz}, 3 \mathrm{H}), 7.15-7.13(\mathrm{~m}, \mathrm{~J}=1 \mathrm{~Hz}, 2 \mathrm{H}), 5.10(\mathrm{~s}, 2 \mathrm{H}), 4.12(\mathrm{~s}, 2 \mathrm{H}), 3.61-3.41(\mathrm{~m}, \mathrm{~J}=10 \mathrm{~Hz}, 4 \mathrm{H}), 3.32-3.12$ $(\mathrm{m}, \mathrm{J}=10 \mathrm{~Hz}, 4 \mathrm{H}) 2.41(\mathrm{~s}, 3 \mathrm{H}) ;{ }^{13} \mathrm{C}$ NMR $\left(75 \mathrm{MHz}, \mathrm{CDCl}_{3}, \delta \mathrm{ppm}\right)=163.70,146.49,145.03,142.18$, $135.13,133.78,129.47,129.00,128.32,127.22,120.07,109.47,52.95,50.96,35.96,13.29$; IR (KBr) $v_{\max }=1659(\mathrm{C}=\mathrm{O}), 1536(\mathrm{~N}=\mathrm{O}), 3125(\mathrm{C}=\mathrm{C}), 2912(\mathrm{C}-\mathrm{C}) \mathrm{cm}^{-1}$; ESI-MS $(\mathrm{m} / \mathrm{z}): 344.1[\mathrm{M}+1]^{+}$; Anal. Calcd for $\mathrm{C}_{17} \mathrm{H}_{21} \mathrm{~N}_{5} \mathrm{O}_{3}$ : C 59.46, H 6.16, N 20.40; Found: C 59.10, H 6.42, N 20.65\%

\section{RESULTS AND DISCUSSION}

The different substituted 2-chloro-1-(piperazin-1-yl)ethanones (J1-J11) were synthesized by aromatic nucleophilic substitution of substituted piperazines on readily available chloroacetyl chloride in good yield. The synthesis of 2-(2-methyl-5-nitro-1H-imidazol-1-yl)-1-(4-phenylpiperazin-1-yl)ethanones (NJ1NJ11) was obtained by reported methods ${ }^{7}$ (Scheme-1). The acetamides emerged as final compounds (NJ1- 
NJ11), which was due to the nucleophilic substitution of commercially available 2-methyl-5nitroimidazol. Aprotic solvent, being DMF, undergoes $\mathrm{SN}_{2}$ inversion by stereochemical evidence that changes in configuration. Potassium iodide (KI) as a catalyst, potassium carbonate $\left(\mathrm{K}_{2} \mathrm{CO}_{3}\right)$ being a weak base, needs the reaction to be refluxed that is heating with the condenser. The reaction undergoes drastic conditions and yield is affected. All the compounds were soluble in $\mathrm{MeOH}$, EtOH, DCM, DMF, DMSO and were recrystallized using appropriate solvent and were stable in the solid-state at room temperature.

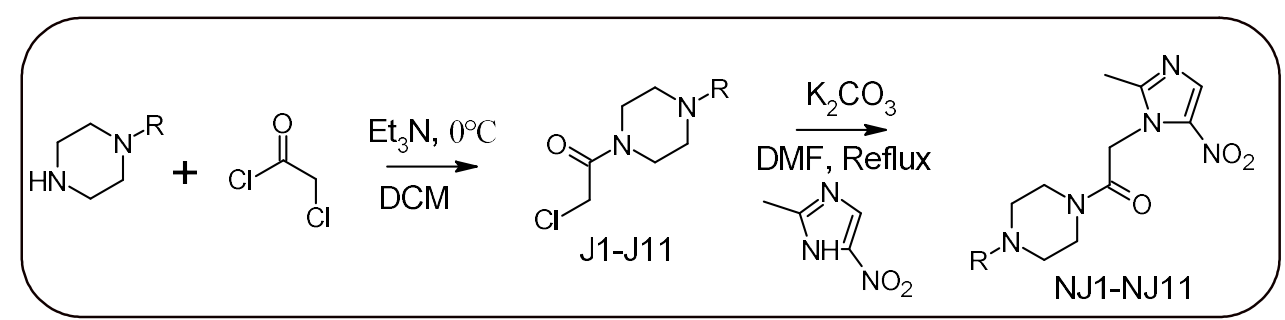

Scheme-1: A Proposed Route for the Synthesis of Final Compounds (NJ1-NJ11)

\section{Cytotoxicity Activity}

Cytotoxicity of synthesized compounds (NJ1-NJ11) has been investigated along with the support of MTT assay as described earlier ${ }^{8,9}$. In brief, nearly 9000-10000 cells/well were placed in a 96-well plate and allowed to grow for 12 hours. After 12 hours, cells were reacted with higher concentrations $(0-200 \mu \mathrm{M})$ of synthesized compounds for 48 hours at $37^{\circ} \mathrm{C}$ in a $\mathrm{CO}_{2}$ incubator. After 48 hours, the medium of cells was aspirated and cells were rinsed two times with phosphate buffer saline ( $\mathrm{pH}$ 7.4). Following the washing, MTT $(20 \mu$ of MTT solution from $5 \mathrm{mg} / \mathrm{ml}$ stock solution in PBS) and $100 \mu \mathrm{l}$ of DMEM were added to each well and plates were incubated additionally for $4-5$ hours at $37^{\circ} \mathrm{C}$ in the $\mathrm{CO}_{2}$ incubator. After $4-5$ hours, incubation, the cell supernatant was aspirated without disturbing any purple color crystals of formazan yielded via reduction of MTT were then absorbed by adding $150 \mu 1$ DMSO. The absorbance was then measured at $570 \mathrm{~nm}$ on a multiplate ELSIA reader (BioRad). The Percentage of cell usefulness has been determined by comparing the treated cells with non-treated ones (control cells) and plotted as a function of the concentration of the compound. As the compounds are DMSO soluble, so to calculate actual cell death, respective concentrations of DMSO has been taken for the treatment of cell and subtracted from the compound treated values.

\section{Cell Viability Assay (MTT)}

The cytotoxicity of these synthesized compounds has been evaluated on HEK293 cells with the help of MTT assay. The cytotoxicity of each compound was studied in a $0-200 \mu \mathrm{M}$ concentration range, for 48 hours. It has been observed that the clinical action of these compounds shows a variable toxicity profile for HEK293 cells. The results of cytotoxicity showed that in the tested concentration range, compound NJ3, NJ5, NJ8 and NJ10 shows considerable cytotoxicity towards HEK293 cells, the respective IC $_{50}$ values of these compounds are shown in Table-1.

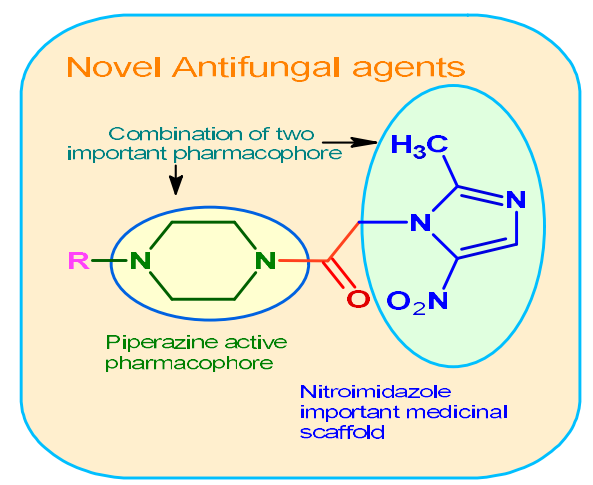

Fig.-1: Diagrammatic Model of Compounds

Whereas other compounds never altered the relevance of HEK293 cells remarkably, as nearly $70 \%$ of cells are active at $200 \mu \mathrm{M}$ concentration of each compound. It has been observed that four of the 


\section{RASĀYAN J. Chem.}

Vol. 14 | No. 4 |2761-2766| October- December | 2021

synthesized compounds are toxic (NJ3, NJ5, NJ8 and NJ10), some are less toxic towards normal human cells (HEK) in the tested concentration range.

Table-1: Cytotoxicity Profile of Synthesized Compounds on HEK293 Cells

\begin{tabular}{|c|c|c|c|}
\hline S.No. & Name of Compound & IC50 $(\mu \mathrm{M})$ & Structure \\
\hline 1. & NJ1 & $>200$ & \\
\hline 2. & $\mathrm{NJ} 2$ & $>200$ & \\
\hline 3. & NJ3 & $175 \pm 4.22$ & \\
\hline 4. & NJ4 & $>200$ & \\
\hline 5. & NJ5 & $102 \pm 5.12$ & \\
\hline 6. & NJ6 & $>200$ & \\
\hline 7. & NJ7 & $>200$ & \\
\hline 8. & NJ8 & $147 \pm 3.45$ & \\
\hline 9. & NJ9 & $>200$ & \\
\hline 10. & NJ10 & $184 \pm 4.23$ & \\
\hline 11. & NJ11 & $>200$ & \\
\hline
\end{tabular}

\section{CONCLUSION}

The present study indicated that the complex consisting of 2-methyl-5-nitroimidazole and piperazine substituted derivatives showed results of cytotoxicity that in the tested concentration range compound NJ3, NJ5, NJ8 and NJ10 show the considerable cytotoxicity towards HEK293 cells. The final compounds (NJ1-NJ11) have been found to possess encouraging structure, which can be put under intensive research to yield biologically active compounds.

\section{ACKNOWLEDGEMENT}

The authors Jyoti Gupta and Afreen Inam are thankful to University Grants Commission, New Delhi, India, for Financial Assistance.

\section{REFERENCES}

1. M. Castellia, M. Malagolia, A. I. Rubertoa, A. Baggioa, C. Casolarib, C. Cermellib, M. R. Bossac, T. Rossid, F. Paoluccie and S. Roffiae, Journal of Antimicrobial Chemotherapy, 40, 19(1997) https://doi.org/10.1093/jac/40.1.19

2. P. Cozzi, U. Branzolli, G. Carganico, C. Ferti, A. Pillan, D. Severino and R. Tonani; European Journal of Medicinal Chemistry, 26, 423(1991) https://doi.org/10.1016/0223-5234(91)90103-T 


\section{RASĀYAN J. Chem.}

Vol. 14 | No. 4 |2761-2766| October- December | 2021

3. D. Olender, J. Zwawiak, V. Lukianchuk, R. Lesyk, A. Kropacz, A. Fojutowski and L. Zaprutko, $\begin{array}{lllll}\text { European Journal of Medicinal Chemistry, 44, 645(2009) } & \end{array}$ https://doi.org/10.1016/j.ejmech.2008.05.016

4. J. D. Firth, P. Brien and L. Ferris, Journal of American Chemical Society, 138, 651(2016) https://doi.org/10.1021/jacs.5b11288

5. R. D. Taylor, M. MacCoss and A. D. G. Lawson, Journal of Medicinal Chemistry, 60, 1638(2017) https://doi.org/10.1021/acs.jmedchem.6b01367

6. P. Chaudhary, R. Kumar, A. K. Verma, D. Singh, V. Yadav, A. K. Chillar, G. L. Sharma and R. Chandra, Bioorganic \& Medicinal Chemistry, 14, 1819(2006) https://doi.org/10.1016/j.bmc.2005.10.032

7. A. Inam, R. L. Van, N. J. Vuuren, C. T. Chen, F. Avecilla, S. M. Agarwal and A. Azam, RSC Advances, 5, 48368(2015), https://doi.org/10.1039/C5RA05472A

8. A.Queen, P.Khan, D.Idrees, A.Azam and M.I.Hassan, International Journal of Biological Macromolecules, 106, 840(2018), https://doi.org/10.1016/j.ijbiomac.2017.08.082

9. M. Voura, P. Khan, S. Thysiadis, S. Katsamakas, A. Queen, G. M. Hasan, S. Ali, V. Sarli and M. I. Hassan, Scientific Reports Nature, 9, 1676(2019), https://doi.org/10.1038/s41598-018-38217-8

[RJC-5943/2020] 